\title{
Revista

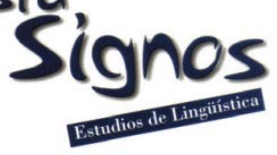

\section{La efectividad del feedback correctivo escrito en la adquisición de segundas lenguas}

\author{
The effect of written corrective feedback in second language \\ acquisition
}

Jorge Lillo Durán

UNIVERSIDAD CATÓLICA DE LA SANTÍSIMA CONCEPCIÓN CHILE

jlillo@ucsc.cl
Katia Sáez Carrillo

UNIVERSIDAD DE CONCEPCIÓN CHILE

ksaez@udec.cl

Recibido: 15-IX-2015 / Aceptado: 30-IX-2016

\section{Resumen}

El feedback correctivo (FC) escrito ha sido un tema polémico en adquisición de segundas lenguas principalmente por los cuestionamientos acerca de su efectividad (Truscott, 1996). El estudio informado en este artículo intenta entregar evidencia de que el feedback correctivo escrito en tres de sus modalidades, a saber, corrección directa sin información metalingüística, con explicación metalingüística y mediada por computador, es efectivo en un contexto de aprendizaje de inglés como L2. El estudio utilizó un diseño cuasi experimental de clases intactas con pretest, postest y postest diferido, comparando el efecto de los tres tipos de feedback sobre la precisión gramatical con que 73 estudiantes de $7^{\circ}$ año básico de un colegio bilingüe español-inglés usaron el pasado simple y la concordancia sujeto verbo. Los datos fueron procesados mediante ANOVA de medidas repetidas con pruebas post hoc de comparaciones múltiples. Los resultados sugieren que el feedback correctivo, en sus tres modalidades, es beneficioso para el proceso de adquisición de las estructuras tratadas en el proceso de producción de textos escritos en inglés como L2 en el contexto de aprendizaje de los participantes, a corto y largo plazo. Además, se encontró que el feedback que entregó información metalingüística escrita fue más consistente en todas las mediciones utilizadas.

Palabras Clave: Feedback correctivo escrito, adquisición de segundas lenguas, errores gramaticales. 


\begin{abstract}
Written corrective feedback (CF) has been a controversial issue regarding its effectiveness in L2 acquisition. The study reported in this article attempts to provide evidence that three types of written corrective feedback, namely, without metalinguistic information, with metalinguistic explanation and computer mediated are effective in a context of English learning as an L2. The study used a quasi-experimental design with intact classes and pretest, post-test and delayed post-test; comparing the effect of the types of feedback on the grammatical accuracy with which 73 students of 7 th grade of a Spanish-English bilingual school used the past simple and subject verb agreement. Data were processed with repeated measures ANOVA, with post hoc multiple comparison tests. The results suggest that the three types of corrective feedback were equally beneficial for the acquisition of the features, both short and long term, in the process of writing in English as an L2 in the learning context of the participants. In addition, it was found that the feedback that provided metalinguistic information was more consistent in all measurements.
\end{abstract}

Key Words: Written corrective feedback, second language acquisition, accuracy.

\title{
INTRODUCCIÓN
}

La escritura en una segunda lengua (L2) es una tarea única, compleja y desafiante, y por consiguiente, la búsqueda de cómo ayudar a los estudiantes a desarrollarla se ha convertido en un objetivo importante para los profesores e investigadores de adquisición de segundas lenguas (ASL). Una de las formas más tradicionales de hacerlo ha sido mediante la entrega de feedback, el que puede cubrir diversos aspectos de escritura como el contenido, la organización textual, la aplicación de las convenciones de los tipos de textos utilizados y el sistema lingüístico. El feedback que específicamente se concentra en el uso de la lengua ha sido denominado feedback correctivo (FC) escrito (Hyland \& Hyland, 2006; Ellis, Sheen, Murakami \& Takashima, 2008; Van Beuningen, 2010) o corrección de la gramática (Truscott, 1996), el tipo de feedback que se preocupa de la respuesta incorrecta o agramatical (Sheen, 2011) en los textos escritos en una L2.

El rol que el FC escrito puede tener en ayudar a los hablantes a mejorar la precisión gramatical de sus textos escritos ha sido tema de debate para investigadores y profesores, porque ha existido divergencia acerca de la efectividad de la corrección de los errores, desde aquellas posiciones que indican que los estudiantes no lo necesitan para progresar (Krashen, 1982), que no es efectivo e incluso perjudicial para la adquisición de la lengua (Truscott, 1996) y aquellos que proponen que el feedback juega un papel preponderante en el aprendizaje de la lengua (Long, 1996; Lyster \& Ranta, 1997; Ellis, 2009; Sheen, 2011), ya que impulsa a los estudiantes a 'notar' e incluso a producir las estructuras lingüísticas de manera que puedan reparar sus errores. A pesar de aquella discrepancia, el tema ha sido objeto de investigación durante las dos últimas décadas, lo que ha promovido exponencialmente los estudios en torno a la gran pregunta en feedback correctivo escrito; es decir, si éste contribuye o 
no a la adquisición de segundas lenguas (Bitchener \& Ferris, 2012; Kang \& Han, 2015). Actualmente, la efectividad del FC escrito ha sido demostrada en algunos casos puntuales en las que las condiciones de investigación empírica han sido controladas en forma rigurosa (Sheen, 2011; Bitchener \& Ferris, 2012; Lillo, 2014) y aunque la gran pregunta todavía está latente, los estudios han derivado en sub cuestionamientos, lo que ha hecho profundizar en temas como la calidad, efectividad, la pertinencia, metodología e interpretación de resultados, entre otros. Los enfoques de investigación se han especializado en observar la problemática desde puntos de vistas específicos y desde esta perspectiva, el estudio informado se fundamenta en tres de ellos; a saber, la investigación en ASL con rigurosidad metodológica y resultados empíricos robustos; los estudios de composición escrita que se centran en habilidades escritas tales como la revisión y la edición textual en una L2 en contextos de aprendizaje real y la investigación acerca de las innovaciones tecnológicas y su impacto en el feedback y especialmente en el feedback correctivo en el aprendizaje de una L2.

En la actualidad, los profesores de segundas lenguas se esfuerzan por ayudar a sus estudiantes a escribir con mayor efectividad, sin embargo, los estudiantes no logran progresar en la producción de textos con mayor precisión gramatical (Ferris, 2010). En el contexto local Chileno, los estudiantes que aprenden inglés como lengua extranjera cometen errores de diferentes característica morfosintácticas, dos de las estructuras gramaticales que presentan dificultades de adquisición para los hablantes son el pasado simple y la concordancia sujeto-verbo.

Existen razones teóricas, empíricas y pragmáticas importantes para considerar estas estructuras gramaticales como problemáticas en su adquisición (Dekeyser, 2007), pero también factibles de ser sometidas a tratamiento de FC. A partir de lo anterior, el propósito del estudio fue determinar si un tratamiento de feedback correctivo escrito que es dado de manera directa (en forma explícita) y focalizada (tratamiento de ambas estructuras), en tres modalidades (corrección directa sin información metalingüística, corrección directa con información metalingüística escrita y corrección directa con información metalingüística escrita y mediada por computador), mejora la precisión gramatical en el uso del pasado simple y la concordancia sujeto-verbo en la producción escrita de textos nuevos en inglés como L2 a corto y largo plazo. El estudio cauteló que las estrategias de FC fueran comparadas con una intervención con tareas de producción escrita idénticas, bajo las mismas condiciones de aplicación, pero en la que no se entregó feedback correctivo escrito, solamente un comentario general sobre el contenido del texto. Los hallazgos sugieren que bajo condiciones experimentales bien controladas, el feedback correctivo escrito en las tres modalidades investigadas promueve la adquisición de las estructuras tratadas. 


\section{Marco teórico}

El feedback correctivo es considerado como una reacción del profesor que invita al estudiante a poner atención a la precisión gramatical de algo que se ha dicho o escrito (Sheen, 2011); esta definición releva los roles de quienes participan en el proceso de feedback, la función orientadora o indicativa del profesor y la de ser capaz de 'notar'1 del estudiante en torno a la precisión gramatical o accuracy. En cuanto al propósito de dar feedback, esto puede variar substancialmente dependiendo, por ejemplo, de la modalidad de la lengua; es decir, si el feedback se da en forma oral o en forma escrita. El primero es una técnica de focalización en la forma (Long, 1996) que apunta a los errores de los estudiantes acerca de su producción en una actividad comunicativa oral (Sheen, 2011), es inmediato y se realiza mediante una comparación en línea; en cuanto al segundo, es el que se entrega acerca de los errores que cometen los estudiantes en sus textos escritos (Bitchener \& Ferris, 2012), no es inmediato y por lo tanto la comparación se realiza en forma diferida. Mientras la mayoría de la investigación en feedback correctivo oral se ha centrado en la corrección de los errores de forma, con el exclusivo propósito de mejorar la precisión gramatical de elementos lingüísticos focalizados, los estudios de FC escrito han tenido una atención variada, distinta a solamente mejorar la precisión gramatical de los textos escritos, porque además se han encargado de mejorar la calidad mediante el desarrollo de estrategias de revisión del contenido y la organización. Esta variación puede explicar por qué la investigación en FC escrito no ha logrado demostrar fehacientemente su efectividad.

\subsection{Efectividad del feedback correctivo en la adquisición de segundas lenguas}

Desde los inicios de la investigación en ASL, los estudios de FC escrito buscaron respuesta a diversas interrogantes en torno al FC; sin embargo, no se concentraron en la macro pregunta en esta temática; es decir, si el FC tiene el potencial de contribuir al desarrollo de la L2 (Bitchener \& Ferris, 2012). En 1996 Truscott en un artículo de gran trascendencia “The Case Against grammar correction in L2 Writing Classes" postuló que la entrega de feedback correctivo escrito no era efectivo y que además podía ser dañino para el desarrollo de la escritura, argumentando que, primero, no existía evidencia empírica para sustentar el supuesto de que la corrección de los errores escritos ayuda a los hablantes de una segunda lengua a mejorar su precisión gramatical, agregando que desde un punto de vista teórico, la corrección de los errores no puede contribuir al desarrollo de la competencia de una L2 o influir en el orden natural y la secuencia de adquisición. Sin embargo, Ferris (1999) argumenta que la base de investigación a la que Truscott hace referencia es demasiado limitada y conflictiva en sus resultados, por lo tanto debiera haber cierta moderación en los comentarios hasta demostrar mayor consistencia en la investigación. También agrega Ferris (1999) que Truscott (1986) habría subestimado el potencial de la evidencia arrojada por algunos estudios en el efecto positivo de la corrección de la gramática y agrega que la 
necesidad de corrección de los estudiantes no se puede desechar u omitir tan fácilmente. Truscott (1996) y Ferris (1999) han coincidido en un punto importante, a saber, que la investigación de la corrección de los errores en la producción escrita en una L2 es insuficiente. A partir de aquello, diversos enfoques de investigación se han especializado en observar la problemática desde puntos de vistas específicos.

Para los investigadores de ASL, la pregunta de mayor relevancia es si el FC escrito puede tener un rol en el procesos de adquisición de una L2 (Bitchener \& Ferris, 2012). Previo al postulado de Truscott (1996) acerca de la inefectividad del FC escrito, nadie había discutido el supuesto ampliamente difundido acerca de la eficacia de corregir los errores escritos de los hablantes de una segunda lengua. La importancia entonces del artículo de Truscott (1996) radica en la fuerza y el impacto acerca de la importancia de la investigación que concierne al rol que pueda o no tener el FC escrito en el proceso de ASL. La evidencia empírica inicial es bastante exigua y con limitaciones metodológicas (Bitchener, 2008). Para poder responder la pregunta acerca de la efectividad del FC escrito, es importante que los estudios sean diseñados apropiadamente, y la literatura indica que un gran número de estudios carecen de un diseño de investigación robusto (Bitchener, 2008; Bitchener \& Ferris, 2012). Algunas de las fallas en el diseño y deficiencias en su ejecución explican hasta qué punto los hallazgos pueden ser calificados como aceptables o no: (1) la falta de un grupo control, (2) falencia para medir la precisión gramatical en textos escritos nuevos y (3) ausencia de formas de medición longitudinal del aprendizaje están dentro los temas más críticos encontrados. Bitchener (2008) ha revisado un gran número de estudios acerca de los efectos del FC escrito, señalando que muy pocos de ellos han incluido un grupo control por lo que no ha sido posible comparar entre aquellos estudiantes que reciben y los que no reciben FC. Adicionalmente, los estudios revisados tenían otras falencias metodológicas, no fueron capaces de demostrar que las ganancias resultantes del FC podían ser aplicadas a la composición de textos nuevos (Truscott \& Hsue, 2008), solo fueron capaces de demostrar que el FC escrito era capaz de ayudar a los estudiantes a mejorar la precisión gramatical en un nuevo borrador de un texto, que es una revisión del texto original. Una última cuestión que es necesaria considerar al revisar los diseños de los estudios, se refiere a la manera en que se mide el aumento en el aprendizaje. Bitchener (2008) recomienda primero establecer el nivel de desempeño de un estudiante, esto generalmente se logra a través de un pre-test de escritura que resalta alguna estructura lingüística que se quiera investigar; de este modo, el mejoramiento de la precisión gramatical, como resultado de la aplicación de FC escrito puede ser medido si otro texto (pos-test), es escrito inmediatamente después que el FC escrito ha sido entregado. Para determinar la estabilidad de los resultados y la efectividad a largo plazo (indicador de adquisición), es necesario además aplicar uno o más pos-test diferidos, dado que los niveles de precisión gramatical pueden ser comparados con los del pos-test y el pre-test. Estos son los temas de diseño más críticos 
de los estudios que han investigado la eficacia del FC escrito. Pero además, existe un rango adicional de problemas, que incluye las deficiencia de ejecución y de análisis de resultados, que han sido identificados por la literatura especializada (Bitchener \& Ferris, 2012).

\subsection{Tipos de FC escrito}

La investigación que ha estudiado la eficacia relativa del $\mathrm{FC}$ escrito se ha concentrado principalmente en los tipos de FC escrito durante los últimos 30 años (Hyland, 2010; Bitchener \& Ferris, 2012). El feedback correctivo directo puede ser definido como la provisión de la forma correcta o la estructura lingüística por encima o cerca del error lingüístico (Van Beuningen, 2010), éste puede incluir el tachar una palabra, frase o morfema que no sea necesario, la inserción de una palabra, frase o morfema o la provisión de la forma correcta. Otras formas de FC directo pueden ser una explicación metalingüística escrita ${ }^{2}$ o una explicación metalingüística oral ${ }^{3}$ (Bitchener, 2008). Por otra parte, el feedback correctivo indirecto señala que de alguna manera, un error ha ocurrido, pero no entrega una corrección explícita. Este puede ser proporcionado subrayando el error, registrando al margen la cantidad de errores en una línea determinada o utilizando un código para mostrar que el error ha ocurrido y qué tipo de error es (Sheen, 2011). El valor del enfoque indirecto radica en el hecho que requiere que los estudiantes participen en aprendizaje guiado y resolución de problemas. Algunos teóricos estiman que el FC directo es preferido por profesores y alumnos, además sugieren que el FC directo reduce el tipo de confusión que resulta cuando los estudiantes no logran comprender o recordar los códigos utilizados por el profesor (Chandler, 2003).

Otra distinción que parece relevante en función del estudio realizado, es la distinción entre FC escrito focalizado y no focalizado. Este último corresponde a la práctica habitual de los profesores de escritura quienes corrigen todos los errores en los trabajos de los estudiantes (Ellis, 2009). Por otra parte, el FC focalizado selecciona errores específicos para ser corregidos y descarta otros. Un FC altamente focalizado se va a concentrar en una, a lo más dos categorías. De alguna manera un feedback correctivo intermedio se puede concentrar en más de dos categorías, pero siempre restringiéndose a un grupo limitado de ellas. Según Ellis (2009), existen razones teóricas solidas que avalan el argumento de que el FC focalizado puede ser más acertado que el FC no focalizado. Los estudiantes están más dispuestos a poner atención a correcciones dirigidas a uno o a un número limitado de tipos de errores y más propensos a desarrollar mayor comprensión de la naturaleza del error y la corrección necesaria. Si la atención (noticing) y el entendimiento (Schmidt, 2001) son necesarios para la adquisición, entonces el FC focalizado está claramente en mejores condiciones para producir mejores resultados. 


\subsection{El feedback correctivo en el contexto de escritura en una L2}

En esta línea de investigación existen estudios que han sido llevados a cabo en contextos de aprendizaje diferentes y con propósitos distintos, a saber, aquellos en que el FC escrito se entrega en contextos de composición de textos con el propósito de desarrollar la escritura en L2 a largo plazo. Como ya se ha comentado, antes de la mitad de la década de los 90, la investigación empírica sobre los efectos del FC escrito era relativamente exigua, una de las razones ha sido el resultado de las corrientes teóricas e históricas imperantes; en primer lugar, la escritura no era importante en la instrucción de la L2 y más tarde fue fuertemente influida por la pedagogía de la escritura de proceso y también por las teorías de ASL de Krashen (1982); en ambos casos, relegando al FC escrito a un papel de menor importancia. Unos pocos estudios se llevaron a cabo entre 1976 y 1996 (Semke, 1984; Robb, Ross \& Shortreed, 1986; Kepner, 1991), los que tuvieron problemas importantes, como los metodológicos, que ya han sido mencionados en el acápite anterior. Posteriormente, una serie de materiales diseñados para abordar las cuestiones lingüísticas en la escritura de una L2 comenzó a aparecer en la década de los noventa, tales como manuales de edición para el inglés como segunda lengua (Lane \& Lange, 1993). Estos autores trataron de evitar los excesos anteriores en la consideración de los errores gramaticales, sin dejar de lado los problemas de precisión gramatical, pero se abogó por que la corrección del error se contextualizara dentro del proceso de escritura recursiva, con prioridad en patrones graves y frecuentes del error escrito y enfocado en las necesidades específicas del estudiante. En ese escenario, en los 20 años transcurridos desde la publicación del artículo original de Truscott (1996), el FC escrito se ha visualizado como una herramienta hacia ese objetivo más amplio y no como un fin en sí mismo.

\subsubsection{Efectos del FC escrito en las habilidades de revisión y edición en una L2}

Uno de los cuestionamientos más recurrentes acerca de los efectos del FC escrito es el relacionado con la validez de los hallazgos de los estudios que investigan la revisión de los textos escritos por los estudiantes después de recibir FC, en función de la comprensión de los procesos de aplicación del feedback así como también del desarrollo de la habilidad escrita de los estudiantes (Ferris, 2010). A pesar de las diferencias en estos estudios, en tanto a metodología y a la diversidad en los contextos de aplicación del FC escrito, es posible concluir que los resultados son muy consistentes en cuanto a que el efecto de corregir los errores que los alumnos cometen revisando y reescribiendo el mismo texto escrito son substanciales (Truscott \& Hsu, 2008). Cuando los estudiantes reciben FC escrito en un texto y se les solicita que lo revisen, ellos lo hacen de manera exitosa (Bitchener \& Ferris, 2012); es decir, con una reducción estadísticamente significativa en el número de errores entre un borrador y otro. Sin embargo, para Truscott y Hsu (2008), el FC escrito que se entrega en el proceso de revisión no entrega evidencia que tal intervención ayude a los estudiantes a 
adquirir estructuras lingüísticas, solamente es útil para ayudar a los estudiantes a mejorar un producto escrito particular en un contexto preciso. Para otros investigadores (Ferris, 2010; Van Beuningen, de Jong \& Kuiken, 2012) el rol del FC escrito en las tareas de revisión del mismo texto, genera las condiciones necesarias que facilitan la adquisición a largo plazo. Esto se sustenta en que los procesos cognitivos involucrados al recibir FC y aplicar las reparaciones a las correcciones en la revisión de los textos escritos, puede producir los tipos de cambios graduales no lineales en la habilidad escrita a largo plazo. Al hacer un paralelo con los resultados de la investigación en FC oral, el uptake inmediato que los estudiantes obtienen del FC es comparable a los resultados informados en los estudios de revisión que puede ser también efectivo en el desarrollo lingüístico a largo plazo. Ferris (2010) sugiere un diseño de investigación que combine el enfoque a corto plazo de los estudios de revisión con los de largo plazo de la investigación en ASL.

\subsection{El feedback correctivo desde la perspectiva del aprendizaje de una segunda lengua mediado por computador}

La investigación que se ha discutido hasta el momento se ha centrado en el feedback correctivo como una estrategia que ocurre en una interacción de carácter presencial. Sin embargo, en la era del aprendizaje a distancia por internet y la comunicación móvil, algunos investigadores de ASL están cada vez más interesados en el rol que tiene el FC en la comunicación mediada por computador (CMC). De acuerdo a sus potencialidades (affordances), la tecnología ha demostrado su relevancia en educación y por consiguiente en la enseñanza y aprendizaje de una segunda lengua. Para los profesionales del lenguaje, CMC podría potencialmente responder dos necesidades; por una parte, podría ser el medio a través del cual se produce la enseñanza y/o podría ser un fin en sí misma (Hyland, 2010).

Desde esta perspectiva, los estudios de FC escrito, han estado orientados a investigar la integración de la tecnología en la producción escrita de manera de explorar el potencial interactivo que ésta tiene (Ware \& Warschauer, 2006). La pregunta central es cómo la respuesta mediada por computador podría imitar o incluso mejorar los resultados informados por la investigación acerca de la interacción de pares o tutor-alumno o tutor-grupo clase, en un ambiente presencial. Los estudios han comparado en el diseño, las prácticas conocidas en el aula con las actividades realizadas con o a través del medio electrónico (Ware \& Warschauer, 2006). Algunos de los primeros estudios examinaron la efectividad del FC en un ambiente de aprendizaje de lenguas asistido por computador; Nagata (1993) comparó el feedback entregado por computador con y sin explicaciones metalingüísticas dirigidas a los errores en el uso de estructuras pasivas en japonés, y encontró que el feedback metalingüístico fue más efectivo que el feedback sin comentarios metalingüísticos. DiGiovanni y Nagaswami (2001) demostraron que los estudiantes estaban más centrados en la tarea al proporcionar feedback durante tiempo real, los instructores 
señalaron las ventajas del medio electrónico que incorpora la capacidad de monitorear las conversaciones entre pares e imprimir las transcripciones. Ellos sugieren un efecto indirecto positivo para los estudiantes que pueden sentir que este tipo de supervisión docente es adecuada. En un pequeño estudio de casos, diseñado para examinar la efectividad de trabajar con los textos escritos de los estudiantes para desarrollar consciencia metalingüística, Yuan (2003) mostró como dos estudiantes analizaron las transcripciones de su propia escritura y de esta manera llegaron a estar más atentos a los errores que ellos produjeron cuando escribieron; la modalidad electrónica fue solamente un medio útil para almacenar sus textos escritos para analizarlos posteriormente en las clases presenciales. El estudio de Hewett (2000) exploró el impacto del feedback en las revisiones; ella investigó cómo los estudiantes aplican a la escritura de sus textos lo que aprendieron a través de la conversación con pares, se destaca que el tipo de interacción tuvo un impacto en la revisión; mientras la conversación oral incluyó el desarrollo de ideas abstractas y globales, el feedback electrónico se enfocó en temáticas de escritura más concretas. Razagifard y Razzaghifard (2011) examinaron el feedback correctivo en contextos comunicativos con tecnología y encontraron que los estudiantes que recibieron FC mediado por computador, superaron a aquellos que no recibieron ni un tipo de feedback.

En relación a CMC en el estudio informado, los rasgos de la interacción entre interlocutores y el feedback correctivo a través de comentarios escritos de una aplicación online pueden conformar el contexto ideal para la investigación de los procesos de adquisición de una segunda lengua; a saber, noticing, noticing the gap, pushed output lo que facilita la prominencia visual de las formas debido a la naturaleza de los turnos escritos que permiten mayor tiempo de procesamiento como resultado de su registro más extenso en la pantalla del computador.

Para resumir, el feedback mediado por tecnología, al igual que el feedback correctivo en composición escrita, muestra una gran diversidad metodológica en los diseños. A pesar de aquello, los hallazgos son alentadores, pero todavía poco se conoce acerca de cómo este tipo de feedback difiere del tradicional y si las mismas variables que dan cuenta de la adquisición en un ambiente presencial son relevantes para el aprendizaje en el contexto de la comunicación mediada por computador (Loewen \& Erlam, 2006; Hyland, 2010).

\section{Marco metodológico}

El estudio se diseñó para responder las siguientes preguntas de investigación:

1. ¿Cuál es la efectividad que tiene el FC escrito directo focalizado en la precisión gramatical del uso del pasado simple y de la concordancia sujeto-verbo en el proceso de producción de textos nuevos escritos en inglés como L2, a corto y largo plazo? 
2. ¿En qué medida el tipo de feedback correctivo escrito determina la precisión gramatical del uso del pasado simple y de la concordancia sujeto-verbo en el proceso de producción de textos nuevos escritos en inglés como L2?

\subsection{Diseño de investigación}

El estudio utilizó un diseño cuasi-experimental de clases intactas, las que funcionaron como grupos experimentales en esta investigación - G1 feedback. correctivo directo focalizado sin información metalingǘstica $(\mathrm{N}=18), \mathrm{G} 2$ feedback. correctivo directo focalizado con explicación metalingüística escrita $(\mathrm{N}=19), \mathrm{G} 3$ feedback correctivo directo focalizado con explicación metalingüística escrita y mediada por computador $(\mathrm{N}=18)$ - y $\mathrm{G} 0$ grupo control $(\mathrm{N}=18)$. Los cuatro grupos completaron un pre-test, un pos-test y un pos-test diferido.

En cuanto al tratamiento, los tres grupos experimentales recibieron corrección de los errores en cuatro textos escritos, 2 de los cuales fueron revisiones del mismo texto. Los tres grupos experimentales recibieron corrección directa (explícita) focalizada (dos estructuras), exclusivamente dirigida a errores que involucraban el uso del pasado simple y de concordancia entre sujeto-verbo en la lengua inglesa. Por otra parte, el grupo control sólo recibió un comentario general acerca del contenido de los cuatro textos escritos.

\subsection{Participantes}

95 estudiantes, 46 mujeres y 49 hombres, de tres cursos de $7^{\circ}$ año básico de un colegio particular bilingüe español-inglés de la comuna de San Pedro de la Paz, Concepción, Chile; cuyas edades fluctúan entre 13 y 14 años de edad, participaron del estudio. Todos los estudiantes completaron una etapa de 6 años de inmersión en la lengua inglesa, equivalente a un nivel B1 en el Marco Común Europeo para la enseñanza - aprendizaje de lenguas extranjeras. De la muestra inicial, 15 de los estudiantes fueron seleccionados por sus profesores para participar en dos pequeños estudios piloto, lo que redujo el $\mathrm{n}^{\circ}$ de participantes a 80 sujetos. De este último grupo, 7 estudiantes fueron descartados, principalmente debido a que sobrepasaron los 90 puntos, equivalentes al $90 \%$ de precisión gramatical en el pre-test escrito 4 . Consecuentemente, la muestra final quedó conformada por 73 estudiantes. Para efectos de la asignatura de inglés, por políticas académicas del establecimiento educacional, los estudiantes de $7^{\circ}$ año son distribuidos en cuatro grupos de habilidades lingüísticas mixtas, cautelando que el nivel de desempeño sea el mismo en cada uno de ellos. De manera aleatoria, se asignó una condición experimental a cada uno de los grupos de inglés de $7^{\circ}$ año básico del establecimiento educacional. 


\subsection{Estructuras}

Las estructuras tratadas con FC escrito son:

1. Pasado simple, específicamente el uso correcto de los verbos regulares, irregulares y cópula verbal.

2. La concordancia entre sujeto y verbo de la tercera persona del presente simple.

\subsection{Tratamiento}

Hubo 5 sesiones de tratamiento. Los estudiantes de los cuatro grupos escribieron los mismos textos (artículos de revista), dos textos nuevos y dos revisiones propiamente tal; cada uno de ellos en lecciones separadas. Los estudiantes de los grupos experimentales recibieron FC escrito por parte del investigador para cada uno de los textos escritos y el grupo control un comentario general del contenido. Previo a la escritura de los artículos de revista, se les pidió a los estudiantes que realizaran dos actividades, una individual y otra en pareja, con el fin de elicitar el vocabulario y contenido necesario para poder realizar la tarea escrita. Posteriormente, se les entregó un estímulo para escribir un texto de 200 palabras. Una vez finalizada la tarea escrita, hubo una actividad léxica de seguimiento que debieron realizar en forma individual. El procedimiento para entregar el FC escrito para cada uno de los grupos experimentales fue el siguiente:

a. El investigador corrigió los textos de los grupos experimentales de acuerdo a los procedimientos de feedback correctivo escrito.

b. A la clase siguiente, los estudiantes recibieron las correcciones correspondientes.

c. Se les pidió a los estudiantes que revisaran los errores y las correcciones cuidadosamente.

d. Una vez revisados los textos escritos, el profesor no realizó comentario alguno acerca de los errores y tampoco entregó alguna explicación adicional.

El procedimiento para el grupo control fue el mismo, excepto que los estudiantes no recibieron FC escrito alguno, solamente un comentario general sobre el contenido del texto.

\subsection{Lineamientos de corrección para los grupos experimentales}

Las modalidades de FC escrito directo focalizado que recibieron los grupos experimentales y el no-FC escrito que recibió el grupo control se puede ver en la Tabla 1. 
Tabla 1. Listado de estrategias de FC escrito directo focalizado del presente estudio.

\begin{tabular}{|l|l|}
\hline Grupos & G1_FSM. Recibió FC escrito directo focalizado sin información \\
& metalingǘstica. \\
G2_FM. Recibió FC escrito directo focalizado con explicación & metalingǘstica escrita. \\
& $\begin{array}{l}\text { G3_FMC. Recibió FC escrito focalizado con explicación } \\
\text { metalingǘstica escrita y mediada por computador. }\end{array}$ \\
\hline Grupo Control & $\begin{array}{l}\text { G0_C. No recibió FC escrito, solo comentarios generales del contenido } \\
\text { y organización del texto. }\end{array}$ \\
\hline
\end{tabular}

A continuación se entrega una breve descripción operacional de cada uno de las estrategias mencionadas anteriormente, así como también de los comentarios de contenidos. Los ejemplos corresponden a extractos del trabajo de estudiantes del estudio.

\subsubsection{Feedback correctivo directo focalizado sin información metalingüística}

Esta opción de corrección entregó la forma correcta. Esto se realizó de diferentes formas:

1. Insertando la forma correcta omitida donde corresponda.

ed

- I think it was fantastic when your discover^ the other world in the closet.

2. Tarjando la palabra o morfema incorrecto e insertando la forma correcta doesn't

- He have the ability of independent life

\subsubsection{Feedback correctivo directo focalizado con explicación metalingüística escrita}

En este caso se entregó la forma correcta, acompañada con una explicación asociada con alguna regla gramatical para el uso del pasado simple y la concordancia entre sujeto y verbo del presente simple.

had (1)

Lady gaga is a good example of success, after going through all the pain she when she has $(2)$

was small I think she is a big person and have a big personality.

(1) - Use the past tense to write about something that happened in the past at a specific time. In this case, these irregular verbs don't follow a specific rule to form the simple past.

(2) - In present tenses, if the third person subject (she) is singular, the verb must be singular too and generally adding an $-s$ at the end. 


\subsubsection{Feedback correctivo directo focalizado con explicación metalingüística escrita y mediada por computador}

Esta modalidad consistió en una mini lección personalizada (Bitchener, 2008) en línea, a través de un blog 5 diseñado para tales efectos (ver anexo 1). Durante esta lección 'online', el investigador mostró un extracto con errores de pasado simple y de concordancia sujeto-verbo tomado desde los textos escritos (tarea 1 y 2) por los estudiantes y les consultó si detectaban errores gramaticales y si eran capaces de repararlos. A continuación estableció un diálogo individual a través de la herramienta de comentarios escritos del blog con cada uno de los miembros del grupo para guiarlos a resolver el problema planteado entregando información metalingüística en forma de reglas gramaticales. Posteriormente, les pidió que revisaran la versión digitalizada de sus textos escritos, los cuales fueron corregidos utilizando explicación metalingüística escrita. Una vez terminado el diálogo les dio acceso a un link con práctica controlada para ejercitar el pasado simple y la concordancia sujeto-verbo.

\subsubsection{Comentarios generales de contenido y organización}

El comentario entregado al grupo control se refirió principalmente al contenido y organización textual.

- Ideas explained clearly, but could perhaps have been slightly more developed.

\subsection{Instrumentos}

Para cada sesión de evaluación se administró un pre-test, pos-test y pos-test diferido. Para estos efectos, los estudiantes escribieron una carta. Se pensó en este tipo de texto porque crea las condiciones necesarias para elicitar las estructuras objeto de estudio y está en concordancia con el tipo de tareas escritas que los estudiantes realizan habitualmente de acuerdo a su programa de estudios. Para que los estudiantes escribieran la carta, se les entregó un estímulo escrito que planteaba una situación relacionada con su entorno académico y a la cual debían reaccionar en forma escrita. Para el pre-test, pos-test y el pos-test diferido, los estudiantes escribieron un nuevo texto (carta), de la misma dificultad, extensión y duración.

El pre-test se administró inmediatamente antes de que los estudiantes recibieran la primera tarea de producción escrita. El pos-test se administró el mismo día que los estudiantes recibieron feedback de su último texto escrito. El pos-test diferido se administró aproximadamente 5 semanas después.

\section{Análisis de resultados}

Para analizar los efectos del tratamiento acerca del uso del pasado simple y la concordancia sujeto verbo, se obtuvieron puntajes para el pre-test, pos-test y pos-test diferido. Esto puntajes fueron calculados mediante el análisis de ocasiones obligatorio 
(Ellis \& Barkhuizen, 2005). Todas las ocasiones obligatorias de uso del pasado simple y de la concordancia sujeto verbo fueron identificadas. Cada ocasión fue examinada para ver la correcta aplicación de las estructuras gramaticales tratadas. Además, la omisión de la aplicación de la estructura también fue considerada como error. El puntaje de precisión gramatical fue calculado para cada texto dividiendo el número de aciertos en el uso de las estructuras por el número total de ocasiones obligatorias más el número de aplicación de la estructura en contextos no obligatorios.

$\mathrm{n}$ aciertos

X 100

$\mathrm{n}$ ocasiones obligatorias $+\mathrm{n}$ aplicaciones en contextos no obligatorios

Los puntajes de las tareas de escritura fueron analizados con ANOVA de medidas repetidas (2 grupos $\mathrm{X} 3$ ocasiones) con pruebas de comparación múltiples post hoc con ajuste Bonferroni.

\subsection{Resultados de los tests escritos por grupo para ambas estructuras}

Lo primero que se informa en esta sección son los resultados de los tests escritos para cada uno de los grupos experimentales y el grupo control, en cuanto el conjunto de las estructuras gramaticales.

Tabla 2. Estadística descriptiva para los tests escritos para cada grupo del estudio.

\begin{tabular}{|c|c|c|c|c|c|c|c|}
\hline Grupo & \multirow{2}{*}{} & T0 & & T1 & & T2 & \\
\cline { 3 - 8 } & & $\mathbf{M}$ & DE & M & DE & M & DE \\
\hline G0_C & 18 & 61,0 & 9,9 & 61,6 & 15,1 & 56,9 & 12,2 \\
\hline G1_FSM & 18 & 63,0 & 10,9 & 82,7 & 10,8 & 71,2 & 13,2 \\
\hline G2_FM & 19 & 63,0 & 13,7 & 81,0 & 10,9 & 81,4 & 7,3 \\
\hline G3_FMC & 18 & 60,9 & 12,6 & 78,7 & 13,3 & 79,8 & 10,9 \\
\hline
\end{tabular}

La Tabla 2 muestra la media y la desviación estándar del pre-test, pos-test y pos-test diferido que midió la precisión gramatical de pasado simple y concordancia sujetoverbo para cada grupo del estudio. El gráfico 1 muestra una representación visual del patrón de los resultados. La Tabla 2 y el Gráfico 1 ilustran que todos los grupos obtuvieron un puntaje sobre 60 puntos en el pre-test, y que solo los tres grupos experimentales fueron capaces de incrementar la precisión entre el pre-test y el pos-test diferido. Sin embargo, solo los participantes de los grupos G2_FM y G3_FMC fueron capaces de incrementar la precisión gramatical después del pre-test, y sostener esta ganancia en precisión en el pos-test diferido, ya que el G1_FSM que tuvo un rápido ascenso en el pos-test, también tuvo un fuerte descenso en el pos-test diferido. 


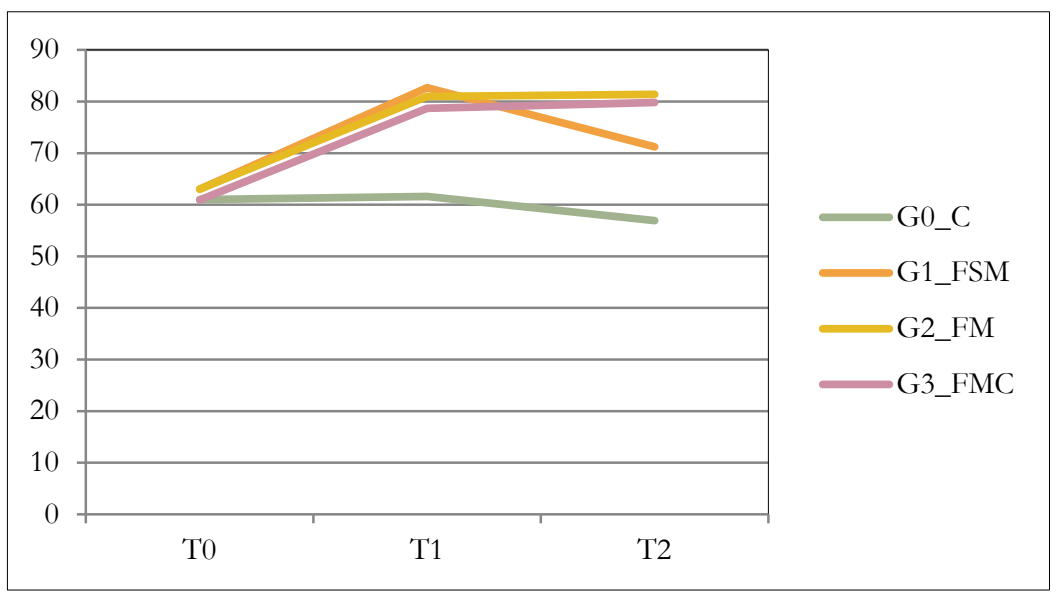

Gráfico 1. Puntajes de los tests escritos de cada uno de los grupos del estudio.

Para poder examinar si las diferencias en los puntajes de los tests escritos en el tiempo eran estadísticamente significativas, se realizó un análisis de medidas repetidas (ANOVA) con la precisión gramatical (puntajes de los tests escritos) como variable dependiente y el tiempo (pre-test, pos-test, pos-test diferido) y el tratamiento de FC escrito como variables independientes.

Tabla 3. ANOVA de medidas repetidas de los puntajes de los tests escritos.

\begin{tabular}{|c|c|c|c|c|c|}
\hline & Origen & $\begin{array}{c}\text { Suma de } \\
\text { cuadrados }\end{array}$ & gl & F & Sig. \\
\hline \multirow{3}{*}{ Prueba intra sujetos } & Tiempo & 3895,667 & 1 & 53,586 &, 001 \\
\cline { 2 - 6 } & Tiempo x Trat. FC & 3218,171 & 3 & 14,756 &, 001 \\
\cline { 2 - 6 } & Error & 5016,282 & 69 & & \\
\hline \multirow{2}{*}{ Prueba inter sujetos } & FC & 7898,158 & 3 & 9,721 &, 001 \\
\cline { 2 - 6 } & Error & 18687,665 & 69 & & \\
\hline
\end{tabular}

Como se puede observar en la Tabla 3 , hubo un efecto significativo para el tratamiento de FC escrito, $F(3,69)=9,721 ; p<, 001$, indicando que los tres grupos del tratamiento, en general, tuvieron un desempeño significativamente mejor que el grupo control en los puntajes totales de los tests. La Tabla 3 también revela evidencia significativa del efecto Tiempo, $F(1,69)=53,586 ; p<, 001$ y de la interacción Tiempo * Tratamiento, $F(3,69)=14,756 ; p<, 001$ indicando que los grupos tuvieron un desempeño diferente en el tiempo.

A la luz de estos resultados, se realizaron diversas pruebas post hoc de comparaciones múltiples (con ajuste Bonferroni con un nivel alfa de 0,05) para aislar exactamente dónde se producían las diferencias. 
Tabla 4. Comparaciones múltiples por grupo.

\begin{tabular}{|c|c|c|c|c|c|c|}
\hline \multirow{2}{*}{ (I)FC } & \multirow{2}{*}{ (J)FC } & $\begin{array}{c}\text { Diferencia } \\
\text { de medias } \\
\text { (I-J) }\end{array}$ & $\begin{array}{c}\text { Error } \\
\text { típ. }\end{array}$ & \multirow{2}{*}{ Sig. } & \multicolumn{2}{|c|}{$\begin{array}{c}\text { Intervalo de confianza al 95 \% para } \\
\text { la diferencia }\end{array}$} \\
\cline { 6 - 7 } & & & & Límite inferior & Límite superior \\
\hline \multirow{3}{*}{ G0_C } & G1_FSM & $-12,5^{*}$ & 3,2 &, 001 & $-21,1$ & $-3,9$ \\
\cline { 2 - 7 } & G2_FM & $-15,3^{*}$ & 3,1 &, 001 & $-23,8$ & $-6,8$ \\
\cline { 2 - 6 } & G3_FMC & $-13,3^{*}$ & 3,2 &, 001 & $-21,9$ & $-4,7$ \\
\hline G1_FSM & G2_FM & $-2,9$ & 3,1 &, 999 & $-11,4$ & 5,6 \\
\hline & G3_FMC &,- 9 & 3,2 &, 999 & $-9,5$ & 7,8 \\
\hline G2_FM & G3_FMC & 2,0 & 3,1 &, 999 & $-6,5$ & 10,5 \\
\hline
\end{tabular}

Estas comparaciones revelaron primero que todo que las diferencias estadísticamente significativas se encontraron entre los puntajes del grupo control y los puntajes de los grupos experimentales. La Tabla 4 revela que, basado en las medias observadas en el estudio en el total de los resultados, los tres grupos experimentales, en forma individual, tuvieron un desempeño superior al grupo control. En cuanto a la comparación entre ellos mismos, no existen diferencia estadísticamente significativas en su desempeño. Como se observa en Gráfico 1, los participantes del grupo uno que recibieron feedback correctivo directo focalizado sin información metalingüística, los del grupo dos que recibieron feedback correctivo directo con explicación metalingüística, así como los del grupo tres que recibieron feedback correctivo focalizado con explicación metalingüística mediada por computador, superaron a los participantes del grupo control que no recibieron feedback correctivo.

Tabla 5. Comparaciones múltiples por tiempo.

\begin{tabular}{|c|c|c|c|c|c|c|c|}
\hline \multirow{2}{*}{ FC } & \multirow{2}{*}{ (I)Tiempo } & \multirow{2}{*}{ (J)Tiempo } & \multirow{2}{*}{$\begin{array}{c}\text { Diferencia } \\
\text { de medias } \\
\text { (I-J) }\end{array}$} & \multirow{2}{*}{$\begin{array}{c}\text { Error } \\
\text { típ. }\end{array}$} & \multirow{2}{*}{ Sig.* } & \multicolumn{2}{|c|}{$\begin{array}{c}\text { Intervalo de confianza al } 95 \\
\% \text { para la diferencia* }\end{array}$} \\
\hline & & & & & & $\begin{array}{l}\text { Límite } \\
\text { inferior }\end{array}$ & $\begin{array}{l}\text { Límite } \\
\text { superior }\end{array}$ \\
\hline \multirow{3}{*}{ G0_C } & \multirow{2}{*}{ T0 } & T1 &,- 6 & 3,1 & ,999 & $-8,1$ & 7,0 \\
\hline & & $\mathrm{T} 2$ & 4,2 & 2,8 & ,442 & $-2,8$ & 11,1 \\
\hline & $\mathrm{T} 2$ & T3 & 4,7 & 2,8 & 286 & $-2,1$ & 11,6 \\
\hline \multirow{3}{*}{ G1_FSM } & \multirow{2}{*}{ T0 } & T1 & $-19,7^{*}$ & 3,1 & ,001 & $-27,2$ & $-12,1$ \\
\hline & & $\mathrm{T} 2$ & $-8,2^{*}$ & 2,9 &, 016 & $-15,1$ & $-1,2$ \\
\hline & $\mathrm{T} 2$ & T3 & $11,5^{*}$ & 2,8 & ,001 & 4,6 & 18,3 \\
\hline \multirow{3}{*}{ G2_FM } & \multirow{2}{*}{ T0 } & T1 & $-18,0^{*}$ & 3,0 & ,001 & $-25,4$ & $-10,7$ \\
\hline & & T2 & $-18,5^{*}$ & 2,8 & ,001 & $-25,3$ & $-11,7$ \\
\hline & $\mathrm{T} 2$ & T3 &,- 4 & 2,7 & ,999 & $-7,1$ & 6,3 \\
\hline \multirow{3}{*}{ G3_FMC } & \multirow{2}{*}{ T0 } & T1 & $-17,7^{*}$ & 3,1 & ,001 & $-25,3$ & $-10,1$ \\
\hline & & $\mathrm{T} 2$ & $-18,9^{*}$ & 2,8 & ,001 & $-25,9$ & $-11,9$ \\
\hline & T2 & T3 & $-1,2$ & 2,8 & (999, & $-8,0$ & 5,7 \\
\hline
\end{tabular}

Al analizar el desempeño de cada uno de los grupos del estudio entre el pre-test (T0), pos-test (T1) y pos-test diferido (T2), las comparaciones múltiples post hoc además demostraron que hubo diferencias estadísticamente significativas para el G1_FSM entre pre-test y pos-test $\mathrm{p}<, 001$; entre el pre-test y el pos-test diferido (T2), $\mathrm{p}=, 016$; así 
como también entre el pos-test y el pos-test diferido $\mathrm{p}<, 001$; lo que indica que la ganancia alcanzada en T1 no se sostiene en T2. Por otra parte, los grupos G2_FM y G3_FMC obtienen diferencias estadísticamente significativas entre pre-test y pos-test, $\mathrm{p}$ $<, 001$; así como también entre pre-test y pos-test diferido, $\mathrm{p}<, 001$; pero no entre pos-test y pos-test diferido, $\mathrm{p}=0,999$, lo que implica que las ganancias alcanzadas por estos grupos en el pos-test, se sostienen en el pos-test diferido.

\section{Discusión}

La primera pregunta de investigación del estudió investigó la efectividad que tiene el FC escrito directo focalizado en la precisión gramatical del uso del pasado simple y la concordancia sujeto-verbo en el proceso de producción de textos nuevos, escritos en inglés como L2, a corto y largo plazo. En este escenario, los resultados de los tests de producción escrita revelaron que, los tres grupos experimentales fueron capaces de incrementar la precisión gramatical entre el pre-test y el pos-test inmediato y entre el pretest y el pos-test diferido, cuando los estudiantes utilizaron el pasado simple y la concordancia sujeto-verbo.

En cuanto al grupo control, que es el grupo que no recibió FC solamente práctica de la producción escrita, los resultados indican que la precisión gramatical de las estructuras no demuestra incrementos. En cuanto a la eficacia de los resultados de los tests de producción escrita, el análisis de medidas repetidas intra sujetos ratificó que las diferencias antes mencionadas, fueron estadísticamente significativas; esto indica que los resultados de los grupos experimentales fue significativamente superior a los resultados del grupo control durante el tiempo en que se llevó a cabo el estudio. Estos resultados al igual que los de Sheen (2007), Bitchener (2008), Ellis et al. (2008) y Sheen, Wright y Moldawa (2009), han demostrado que un tratamiento de FC que ha sido riguroso en el procedimiento metodológico en cuanto a la utilización de un grupo control, a la escritura de textos nuevos y a la medición longitudinal de resultados, tiene efectos importantes en el proceso de adquisición de dos estructuras difíciles de adquirir para hablantes de español que aprenden inglés como L2. Además, es probable que la incorporación de la revisión de textos al diseño haya generado las condiciones necesarias que facilitan la adquisición a largo plazo, lo que se opone a lo informado por Truscott y Hsu (2008) quienes afirman que el FC escrito no afecta la adquisición de la L2, ni la producción de textos escritos nuevos y que solo funciona al recomponer el mismo texto una segunda vez.

La segunda pregunta consistió en averiguar si el tipo de feedback correctivo escrito determina la precisión gramatical del uso del pasado simple y de la concordancia sujeto-verbo, en el proceso de producción de textos nuevos en inglés como L2. Como ya ha sido informado, los resultados generales indican que los grupos experimentales superaron al grupo control, que no fue capaz de mejorar su desempeño en el pos-test 
inmediato y tampoco en el pos-test diferido. Esta visión fue corroborada estadísticamente mediante ANOVA de medidas repetidas que demuestra una diferencia estadísticamente significativa entre grupos (tratamiento de FC); es decir, los grupos experimentales fueron superiores al grupo control cuando el tiempo fue controlado. Las comparaciones múltiples entre los grupos del estudio en relación a los resultados para el conjunto de las estructuras, demostraron diferencias estadísticamente significativas entre cada uno de ellos y el grupo control; es decir, las tres formas de tratamiento de feedback correctivo escrito directo focalizado fueron superiores a la práctica de escritura que no recibió FC, solamente un comentario general de contenido y organización. En esta comparación, los resultados revelan además que no existieron diferencias estadísticamente significativas entre los grupos experimentales, este es un indicador de que todos los tratamientos fueron efectivos. Un segundo análisis de comparaciones múltiples de cada uno de los tratamientos en función de las ganancias obtenidas entre pre-test y pos-test, y entre pre-test y pos-test diferido indican que hubo diferencias estadísticamente significativas para los tres grupos experimentales, no así para el grupo control. En este análisis es interesante observar que el nivel de precisión del grupo 1 fue menor que el de los grupos que además entregaron información metalingüística. El descenso en el desempeño del G1_FSM en el pos-test diferido podría indicar que este tipo de feedback produce un efecto a corto plazo importante, pero no tanto a largo plazo, porque el procesamiento de los errores puede ser menos profundo (Ferris, 2010), ya que la resolución inmediata del problema mediante la entrega de la respuesta correcta, no demanda mayor esfuerzo cognitivo, y por consiguiente el efecto del tratamiento puede no ser tan potente a largo plazo como en aquellos casos en que se entrega información explícita en forma de reglas que demanda mayor reflexión, esfuerzo cognitivo y mayor profundidad de procesamiento. Los hallazgos también contribuyen en relación a estudios previos; primero, corroboran lo informado por Bitchener, Young y Cameron, (2005) quienes encontraron que la adición de explicación metalingüística escrita ayuda a los estudiantes a mejorar la precisión gramatical de su escritura, pero además demuestran que una explicación metalingüística oral a través de una mini-lección focalizada, a través de una aplicación computacional (blog), entregada en forma individual a los estudiantes, puede llegar a ser tan efectiva como una forma de FC tradicional.

\section{CONCLUSIONES}

El estudio informado en este artículo ha investigado la entrega de feedback correctivo escrito directo y focalizado en un contexto escolar que promueve el proceso de producción de textos escritos en inglés como L2. Los casos a favor y en contra del feedback correctivo escrito no se sostienen solamente en lo que la investigación ha logrado descubrir acerca de su efectividad; sin embargo, esta evidencia es un aspecto fundamental que debe ser considerado (Ellis et al., 2008). El 
descrédito de Truscott (1996) por el valor del FC escrito se basa en lo que él ha observado, así como también en la ausencia de evidencia empírica que demuestre que éste contribuye a la adquisición, que se manifiesta en el mejoramiento de la precisión gramatical en textos escritos subsecuentes, y aunque esta evidencia es cada vez mayor, los detractores del FC escrito (Truscott \& Hsu, 2008) consideran que aún la investigación de la última década es limitada e incluso conflictiva en cuanto a sus resultados y procedimientos metodológicos. A partir de aquello, distintas líneas de investigación han aceptado el desafío de demostrar, no tan solo la efectividad del FC de manera general a largo plazo, sino también cómo éste puede ser aplicado en diferentes contextos; como resultado, estudios empíricos con un diseño metodológico robusto (Bitchener et al., 2005; Sheen, 2007; Ellis et al., 2008, Van Beunighen et al., 2012) han demostrado que el FC aplicado a la reparación de errores en el uso de una o dos estructuras gramaticales si tiene un efecto positivo; en forma paralela, estudios cuyo foco ha sido el que los estudiantes aprendan a escribir en una L2, han utilizado el FC de manera que los estudiantes sean capaces de desarrollar estrategias de revisión y edición de textos con resultados positivos a corto plazo, pero no tan concluyentes a largo plazo (Ferris, 2010) y finalmente, estudios de tecnología en el aprendizaje de segundas lenguas han profundizado en cómo las innovaciones tecnológicas pueden impactar positivamente en el sistema completo de feedback y en especial de FC (Razagifard \& Razzaghifard, 2011). Estas líneas de investigación han sido uno de los componentes fundamentales que sostiene el modelo de investigación integrado que se ha implementado en el estudio (Lillo \& Ferreira, 2014); a saber, un modelo robusto metodológicamente, que fomenta la escritura recursiva y que incorpora la tecnología. A partir de esto, el estudio concluye que a diferencia de la mayoría de la investigación en FC escrito que ha sido realizada en contextos de educación superior, el FC escrito es efectivo en ayudar a un grupo de estudiantes escolares de L2 a mejorar la precisión gramatical de dos estructuras a corto y largo plazo, lo que puede ser una indicación de adquisición; además, es probable que el haber realizado revisiones de los textos después de recibir feedback sea un factor importante en la asimilación del FC y que el uptake resultante de este proceso promueva la retención de las estructuras erróneas; el estudio también demuestra que el FC escrito entregado a través de una mini-lección online, componente de un mismo diseño de investigación, puede ser tan efectivo como otras formas de feedback tradicional cuando las condiciones experimentales han sido bien controladas. La utilización de los constructos principales de la teoría sociocultural en la entrega del FC a través de la mediación por computador, en conjunto con constructos cognitivos, permitieron la eficacia en el efecto del FC escrito al mismo nivel de los resultados en situaciones donde prima la visión cognitivista y en modalidad tradicional presencial. Los estudiantes fueron capaces de notar el error y corregirlo, pero con asistencia del tutor experto quien a través de un proceso de andamiaje varió las estrategias específicas que utilizó con los estudiantes, quienes entendieron la ayuda y fueron capaces de incorporar el feedback que se les dio. Otro 
punto importante a destacar es que los estudios que han sido exitosos en proporcionar evidencia de la utilidad del FC escrito se han concentrado principalmente en los artículos del inglés (Sheen, 2007; Ellis et al., 2008; Van Beuningen et al., 2012); muy pocos son los que han explorado otras categorías gramaticales (Bitchener et al., 2005). Claramente, la investigación requiere evidencia que el FC puede afectar positivamente la adquisición de una gama más variada de estructuras gramaticales. Por consiguiente se necesita investigación que apunte a categorías diferentes y en ese sentido, la presente investigación contribuye a la base de estudios empíricos, porque explora cómo el FC escrito es un factor que facilita el aprendizaje y adquisición de otras estructuras gramaticales (pasado simple y concordancia sujeto-verbo) declaradas como poco transparentes (DeKeyser, 2007) y difíciles de adquirir para los hablantes de español que aprenden inglés como L2. Finalmente, los hallazgos del estudio indican que si los estudiantes toman consciencia de la información metalingüística explícita que se les entrega, mayor puede ser la efectividad del feedback, lo que podría ser un indicador de adquisición.

Mucho de la investigación reciente en FC se ha desarrollado en contextos de educación superior y principalmente en el hemisferio norte. Este estudio se llevó a cabo en un contexto de educación escolar, regional. Los resultados son auspiciosos y por consiguiente, es necesario desarrollar investigación a mayor escala en el contexto Chileno que permita descubrir con mayor exactitud cómo nuestros estudiantes aprenden Inglés o alguna otra segunda lengua y cómo se puede contribuir al mejoramiento de su adquisición.

\section{REFERENCIAS BIBLIOGRÁFICAS}

Bitchener, J. (2008). Evidence in support of written corrective feedback. Journal of Second Language Writing, 17(2), 102-118.

Bitchener, J. \& Ferris, D. (2012). Written corrective feedback in second language acquisition. Nueva York: Routledge.

Bitchener, J., Young, S. \& Cameron, D. (2005). The effect of different types of corrective feedback on ESL student writing. Journal of Second Language Writing, 14(3), 191-205.

Chandler, J. (2003). The efficacy of various kinds of error feedback for improvement in the accuracy and fluency of L2 student writing. Journal of Second Language Writing, 12(3), 267-296.

DeKeyser, R. (2007). Practice in a second language: Perspectives from applied linguistics and cognitive psychology. Nueva York: Cambridge University Press.

DiGiovanni, E. \& Nagaswami, G. (2001). Online peer review: An alternative to faceto-face? ELT Journal, 55(3), 263-272. 
Ellis, R. (2009). A typology of written corrective feedback types. ELT Journal, 63(2), 97-107.

Ellis, R. \& Barkhuizen, G. (2005). Analyz̧ing learner language. Oxford: Oxford University Press.

Ellis, R., Sheen, Y, Murakami, M. \& Takashima, H. (2008). The effects of focused and unfocused written corrective feedback in English as a foreign language context. System, 36, 353-371.

Ferris, D. (1999). The case for grammar correction in L2 writing classes: A response to Truscott (1996). Journal of Second Language Writing, 8, 1-10.

Ferris, D. (2010). Second language writing research and written corrective feedback. Studies in Second Language Acquisition, 32, 181-201.

Hewett, B. (2000). Characteristics of interactive oral and computer-mediated peer group talk and its influence on revision. Computers \& Composition, 17, 265-88.

Hyland, F. (2010). Future directions in feedback on second language writing: Overview and research agenda. International Journal of English Studies, 10(2), 171 182.

Hyland, K. \& Hyland F. (2006). Feedback in second language writing: Contexts and issues. Cap. 7. Cambridge: Cambridge University Press.

Kang, E. \& Han, Z. (2015). The efficacy of written corrective feedback in improving L2 written accuracy: A meta-analysis. The Modern Language Journal. 99(1), 1-18.

Kepner, C. (1991). An experiment in the relationship of types of written feedback to the development of second-language writing skills. The Modern Language Journal, 75(3), 305-313.

Krashen, S. (1982). Principles and practices in second language acquisition. Oxford: Pergamon Press.

Lane, J. \& Lange, E. (1993). Writing clearly: An editing guide. Boston, MA: Heinle \& Heinle.

Lillo, J. (2014). Efecto del feedback correctivo escrito directo focalizado, en el proceso de producción de textos escritos en inglés como L2. Tesis de doctorado, Universidad de Concepción, Chile.

Lillo, J. \& Ferreira, A. (2014). Un modelo teórico-metodológico para investigación empírica en feedback correctivo escrito en una segunda lengua. Onomaz̧ein, 30, 90-110. 
Long, M. (1996). The role of the linguistic environment in second language acquisition. Handbook of second language acquisition, 26, 413-468.

Loewen, S. \& Erlam, R. (2006). Corrective feedback in the chatroom: An experimental study. Computer Assisted Language Learning, 19(1), 1-14.

Lyster, R. \& Ranta, L. (1997). Corrective feedback and learner uptake. Studies in Second Language Acquisition, 19, 37-66.

Nagata, N. (1993). Intelligent computer feedback for second language instruction. The Modern Language Journal, 77(3), 330-339.

Razagifard, P. \& Razzaghifard, V. (2011). Corrective feedback in a computer-mediated communicative context and the development of second language grammar. Teaching English with Technology, 11(2), 1-17.

Robb, T., Ross, S. \& Shortreed, I. (1986). Salience of feedback on error and its effect on EFL writing quality. TESOL Quarterly, 20(1), 83-93.

Schmidt, R. (2001). Attention. En P. Robinson (Ed.). Cognition and second language instruction (pp. 1-32). Nueva York: Cambridge University Press.

Semke, H. (1984). Effects of the red pen. Foreign Language Annals, 17(3), 195-202.

Sheen, Y. (2007). The effect of focused written corrective feedback and language aptitude on ESL learners' acquisition of articles. TESOL Quarterly, 4, 255-283.

Sheen, Y. (2011). Corrective feedback individual differences and second language learning. Nueva York: Springster.

Sheen, Y., Wright, D. \& Moldawa, A. (2009). Differential effects of focused and unfocused written correction on the accurate use of grammatical forms by adult ESL learners. System, 37(4), 556-569.

Truscott, J. (1996). Review article. The case against grammar correction in 12 writing classes. Language Learning, 46(2), 327-369.

Truscott, J. \& Hsu, A. (2008). Error correction, revision, and learning. Journal of Second Language Writing, 17(4), 292-305.

Van Beuningen, C. (2010). Corrective feedback in 12 writing: Theoretical perspectives, empirical insights, and future directions. International Journal of English Studies, 10(2), 1-27.

Van Beuningen, C.G., De Jong, N.H. \& Kuiken, F. (2012). Evidence on the effectiveness of comprehensive error correction in second language writing. Language Learning, 62(1), 1-41. 
Ware, P. \& Warschauer, M. (2006). Electronic feedback and second language writing. En K. Hyland \& F. Hyland (Eds.). Feedback and second language writing. Context and issues (pp. 105-122). Cambridge: Cambridge University Press.

Yuan, Y. (2003). The use of chat rooms in an ESL setting. Computers and Composition, 20, 194-206.

\section{NOTAS}

${ }^{1}$ Long (1996) considera que el feedback correctivo es una estrategia esencial de la focalización en la forma porque atrae la atención de los estudiantes hacia los enunciados erróneos de los hablantes. Si el noticing es un constructo fundamental en ASL porque tiene un rol mediador entre el input lingüístico y la adquisición, entonces el feedback correctivo es capaz de acelerar este proceso porque permite al hablante poner atención, notar la forma lingüística, darse cuenta de la brecha que existe con la lengua meta y eventualmente modificar su interlengua.

${ }^{2}$ La provisión de reglas gramaticales y los ejemplos al final de la escritura de un estudiante con una referencia a los lugares en el texto donde él ha cometido un error.

${ }^{3}$ Una mini-lección en la que las reglas y ejemplos se presentan, se practican y se discuten, en una modalidad profesor alumno o profesor con grupos pequeños de alumnos.

4 Ellis et al. (2008) consideran que un porcentaje superior al $90 \%$ es un indicador de adquisición.

${ }^{5}$ Se diseñó un blog individual por estudiante, apelando a que era un medio de comunicación basado en tecnologías bastante familiar para ellos, de fácil acceso y utilización. De todas maneras, esto implicó generar un diseño gráfico en el que fuese presentada la mini lección, los textos escaneados de los estudiantes y la práctica de las estructuras. 


\section{ANEXO}

Feedback correctivo directo focalizado con explicación metalingüística escrita y mediada por computador

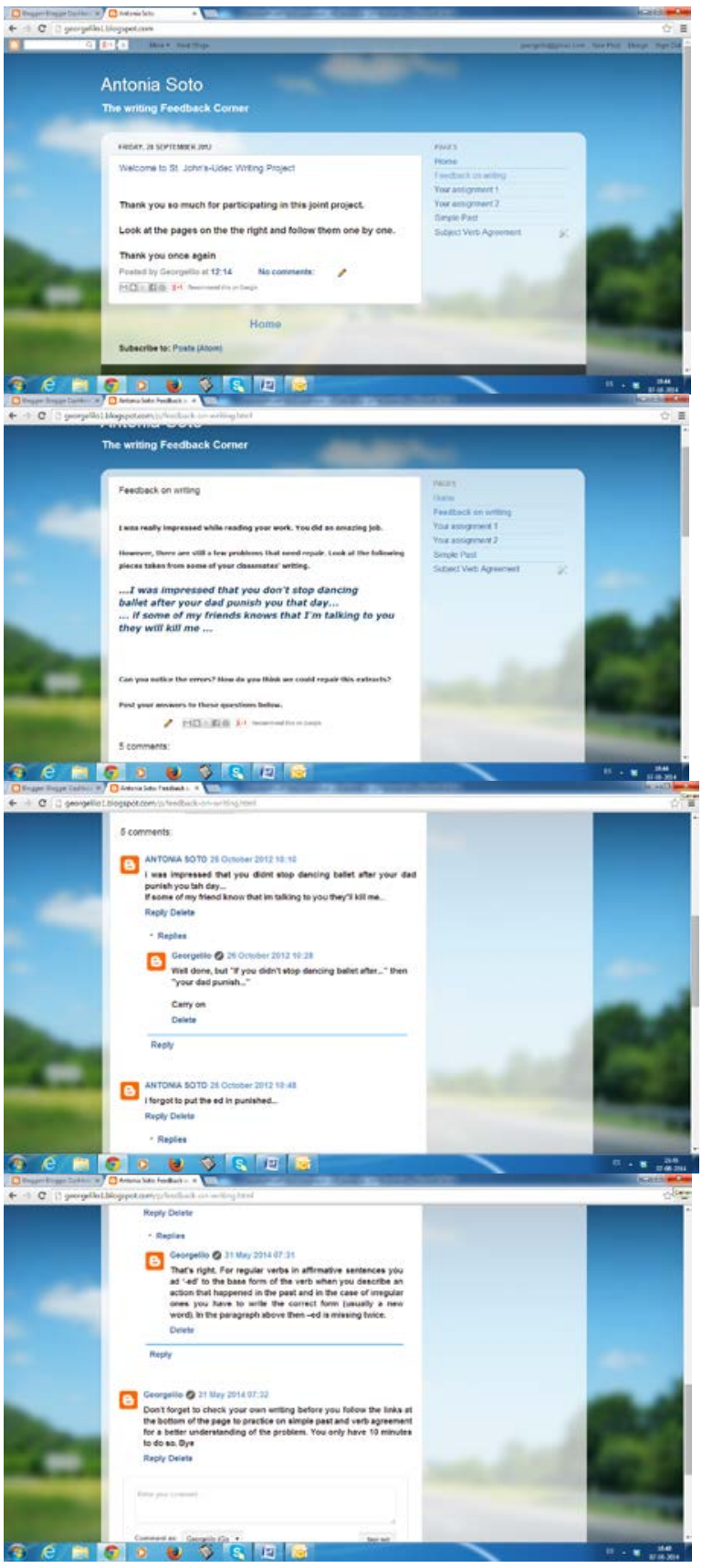

